\title{
Effects of tobacco smoking and abstinence on middle latency auditory evoked potentials
}

\begin{abstract}
Objective: To evaluate the effects of tobacco cigarette smoking and overnight abstinence on middle latency auditory evoked potentials among smokers and nonsmokers.

Methods: Groups of 9 to $\mathbf{1 0}$ adult male and female nonsmokers and smokers participated in the study. Each person volunteered for two laboratory sessions conducted in the early afternoon on 2 separate days. Smokers abstained from tobacco products 6 to 15 hours before the abstinent session and maintained their usual smoking behavior before the smoking session. The nonsmokers had a similar laboratory experience but sham smoked by means of inhaling air. Middle latency auditory evoked potentials were recorded from $\mathrm{Cz}$ to both ears as reference.

Results: The latencies of the $\mathrm{Na}$ and Pa potentials during the smoking session were significantly $(p<0.01)$ shorter than those in abstinent smokers and nonsmokers. After smoking, peak-to-trough amplitudes for the $\mathrm{V}-\mathrm{Na}$, Na-Pa, and $\mathrm{Pa}-\mathrm{Nb}$ potentials were larger than those after abstinence and significantly larger than those among nonsmokers.

Conclusions: The shorter latencies of the middle latency brain wave components in the smoking session suggest faster processing of sensory information after cigarette smoking. Larger $\mathrm{Pa}$ amplitudes after cigarette smoking suggest a higher arousal level than that among partially abstinent smokers and nonsmokers. (Clin Pharmacol Ther 1998;63:571-9.)
\end{abstract}

Takuzo Kishimoto, MD, a and Edward F. Domino, MD Ann Arbor, Mich.

Most tobacco users report that smoking produces mental stimulation, particularly with the first few cigarettes of the day. ${ }^{1}$ Electroencephalographic (EEG) stud-

From the Department of Pharmacology, University of Michigan.

Supported in part by the Psychopharmacology Research Fund 361024 (University of Michigan, Ann Arbor, Mich.) and by grants DA-07226 and DA-10992 from the National Institute on Drug Abuse (Rockville, Md.). Dr. Kishimoto was a clinical pharmacology fellow supported in part by the Japanese Society of Clinical Pharmacology (Tokyo, Japan) during the course of this study.

A preliminary report of this research was presented at the Ninetysixth Annual Meeting of the American Society for Clinical Pharmacology and Therapeutics, San Diego, Calif., March 15-17, 1995 (Kishimoto T, Domino EF. Effects of tobacco smoking and abstinence on short and middle latency auditory evoked potentials (SLAEP and MLAEP) in smokers compared to nonsmokers. Clin Pharmacol Ther 1995;57:160).

Received for publication Sept. 3, 1997; accepted Dec. 4, 1997.

Reprint requests: Edward F. Domino, MD, Department of Pharmacology, A220E MSRB III, University of Michigan, Ann Arbor, MI 48109-0632.

aCurrent address: Department of Psychiatry, Tenri Hospital, 200 Mishima-cho, Tenri City, Nara 632, Japan.

Copyright $\odot 1998$ by Mosby, Inc.

$0009-9236 / 98 / \$ 5.00+0 \quad \mathbf{1 3 / 1 / 8 7 9 5 7}$ ies have shown that nicotine produces cortical activation with a shift to higher EEG frequencies. ${ }^{2-8}$ These changes are associated with arousal and resemble those produced in animals by means of electrical stimulation of the ascending reticular activating system. ${ }^{9}$ Nicotine acts on this system to modify the arousal level. ${ }^{10-14}$ Abstinence from tobacco smoking is accompanied by EEG signs of sedation (decrease in EEG alpha and beta frequencies and an increase in theta frequency). 5,15

Little is known about the effects of nicotine or tobacco smoking on middle latency auditory evoked potentials (MLAEP). The amplitude of Pa of the MLAEP (Fig. 1) has been shown to reflect subjects' state of arousal. ${ }^{16,17}$ Furthermore, injection of lidocaine into the mesencephalic reticular formation of animals produces a reduction of MLAEP, indicating that these auditory evoked potentials (AEP) receive a critical input from this brain region. ${ }^{18}$ The hypothesis of our research was that the amplitude of the $\mathrm{Pa}$ potential would increase immediately after smoking and would be reduced after abstinence, consistent with nicotine's producing more arousal and its absence causing less. These effects of tobacco smoking and abstinence on MLAEP may help to explain some of the motivation smokers have to maintain their smoking behavior. 


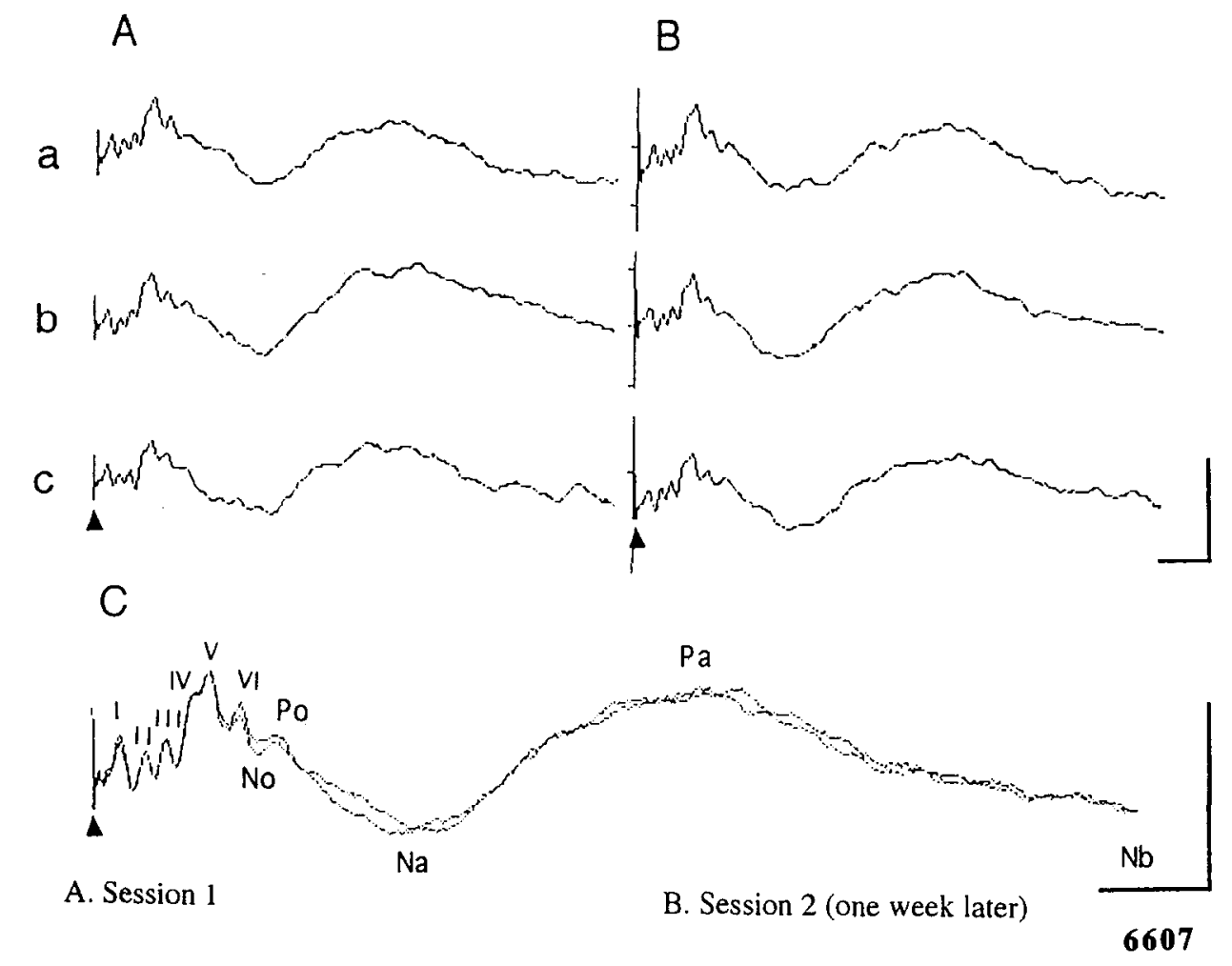

Fig. 1. Reproducibility of middle latency auditory evoked potentials (MLAEP) among nonsmokers recorded in a pair of experimental sessions separated by about 1 week. A, First session. B, Second session. In each session, MLAEP recordings lasting for about 2 minutes were repeated to obtain three reliable responses for each subject $(n=10)$. The potentials from $a, b$, and $c$ represent the global averaged waveforms from the 10 nonsmokers' first, second, and third 2-minute recordings in each session (A and B). These averaged MLAEP were further averaged over the three recordings for each session. C, Superimposed overall mean MLAEP for all 10 nonsmokers. The early components I through VI and three MLAEP Po, Na, and $\mathrm{Pa}$ are labeled. The superimposed tracings are from the first session. The height of the vertical bar is $0.5 \mu \mathrm{V}$, positive up. The length of the horizontal bar is $5 \mathrm{msec}$ in this figure and in Figs. 2 and 4. The two sessions produced similar MLAEP. The triangle represents the onset of the auditory stimulation in this figure and in Figs. 2 and 4.

\section{METHODS}

Subjects. This study was approved by the Committee to Review Clinical Research and Investigation Involving Human Beings of the University of Michigan Medical School. Eleven adult healthy cigarette smokers and 10 nonsmokers were recruited from university staff and students by means of local advertisement. Smokers were required to meet the criteria of having smoked, through inhalation, 15 or more cigarettes per day for at least 1 year. The smokers completed a questionnaire to characterize their smoking behavior before selection for the study. Two of the 11 smokers recruited were excluded from analysis because of an elevated or zero plasma nicotine and cotinine level in both smoking and abstinence sessions as the result of obvious noncompli- ance with instructions. The remaining nine smokers were six men and three women (mean age \pm SEM, 29.0 \pm 2.3 years). Three smokers were white, and six were Asian. The mean \pm SEM number of cigarettes smoked per day was $20.2 \pm 1.5$; duration of smoking was 10.0 \pm 2.5 years. The nonsmokers were seven men and three women (mean age \pm SEM, $30.0 \pm 2.34$ years). Three nonsmokers were white and seven were Asian. Subjects taking concurrent medication or with a history of central nervous system problems, such as schizophrenia, affective or anxiety disorders, and drug abuse, were excluded from the study. Subjects were paid hourly for time spent in the laboratory.

Experimental design. Two laboratory sessions of about 2 hours separated by about 7 days were conducted for each 
smoker and nonsmoker. The pair of sessions for each subject were held in the early afternoons about the same time of day, typically between 1:30 and 3:30 PM. All subjects were instructed to refrain from alcohol and caffcinated beverages for the morning period before both sessions. The probable differential caffcine intake betwcen smokers and nonsmokers was not controlled. One of the two sessions for each smoker was designed so that the subject smoked cigarettes in the morning as usual (smoking session), whereas in the other session the subject was instructed to be abstinent from smoking overnight and through the early afternoon, at least for 12 to 15 hours before coming to the laboratory (abstinent session). In all probability, not all of the abstinent tobacco smokers abstained from smoking overnight through to early afternoon but smoked in the early morning. Therefore the abstinent period was probably 6 to 15 hours. During the smoking session, one would expect the mean plasma concentration of nicotine in most cigarette smokers to reach its steady state in the afternoon. ${ }^{19,20}$ MLAEP measurement was performed after subjects smoked a cigarette in the smoking session. In the abstinent session, the same sets of AEP measurements were performed with the subjects sham smoking air. Expired alveolar air and venous blood were taken to assay carbon monoxide and plasma nicotine and cotinine concentrations at both the beginning and end of the session. Heart rate (electrocardiographic lead II) and arterial blood pressure (by means of auscultation) were measured. The two experimental sessions for each nonsmoker were as similar as possible to those of the smokers, except for the crucial variables of cigarette smoking, abstinence, venous blood withdrawal, and exhaled carbon monoxide measurements.

Procedure. The smokers were asked to smoke their usual number of cigarettes in the morning before coming to the laboratory in the early afternoon for the smoking session. On arrival, each subject's consent was reconfirmed. They were then taken to the recording rom where alveolar carbon monoxide levels were sampled, venous blood withdrawn, and arterial blood pressure and heart rate measured. After electrode placement on the scalp, each subject lay down on a cot, and hearing thresholds to monaural stimulation for the left and right ears were estimated. The first AEP recording was then performed.

An MLAEP recording was performed for approximately 2 minutes and repeated until three reliable MLAEPs were obtained. Before the end of the session, alveolar carbon monoxide and venous blood were again sampled. Arterial blood pressure and heart rate were taken at the end of the session. The subjects repeated inhalation of a cigarette of their own brand for about 4 minutes before each set of three AEPs was recorded. The same procedures and time course were used in the abstinent session as in the smoking session, cxccpt that the subjects stated they had not smoked any cigarettes since the night before. The nonsmokcrs wcre examined in the same manner except for the sampling of alveolar air and venous blood. Experimental times were recorded manually and with an internal computer clock for AEP acquisition.

Auditory evoked potential recordings. The AEP were recorded with silver-silver chloride electrodes placed on vertex $(\mathrm{Cz})$ and referred to linked $\mathrm{A} 1$ and $\mathrm{A} 2$ electrodes with adhesive conductive paste. All electrode impedances were kept below $5 \mathrm{k} \Omega$. For recording MLAEP, auditory stimulation consisted of click tones $(70 \mathrm{~dB}, 0.1 \mathrm{msec}$ duration of alternating polarity) presented every 4.1 seconds to the left ear through the headphones. The electrical signals were amplified with a bandpass set at 5 to $3000 \mathrm{~Hz}$ and a time base of 0 to $50 \mathrm{msec}$. A total of 500 artifactfree sweeps were averaged with version 6.6 software and Nicolet Pathfinder I equipment (Nicolet; Analytical Instruments, Madison, Wis.).

Assay of carbon monoxide and nicotine. The amount of carbon monoxide in the expired air samples was measured. ${ }^{6}$ The assay of plasma nicotine was performed with HPLC. 21

Data analysis. AEP data were stored on diskettes and analyzed off line. MLAEP affected by nonbiologic alteration of the isoelectric line or any other artifact were discarded. Three sequential MLAEP were obtained in each session. These were called the first, second, or third MLAEP. Early short latency peaks I through VI and MLAEP peaks including $\mathrm{Na}, \mathrm{Pa}$, and $\mathrm{Nb}$ were identified by means of visual inspection (Fig. 1). Latencies, interpeak latencies, and peak-to-trough amplitudes of these components were scored with the aid of the Nicolet computer cursor program. The data from each of the three artifact-free MLAEP selected for each subject were averaged across subjects. These values were subjected to correlated Student $t$ tests between the two sessions (smoking and abstinence) for smokers and noncorrelated $t$ tests between smokers and nonsmokers. The waveforms of the MLAEP of each group were averaged to create a global mean waveform for each test group.

\section{RESULTS}

Plasma nicotine, cotinine, and alveolar carbon monoxide concentrations of smokers. With a few exceptions, all smokers had venous blood and expired alveolar air samples taken at the beginning and end of each session. Two of the nine smokers studied who showed somewhat high plasma nicotine and cotinine levels were noncompliant with the instruction to abstain from smoking for at least 12 to 15 hours. ${ }^{22,23}$ Thus the 

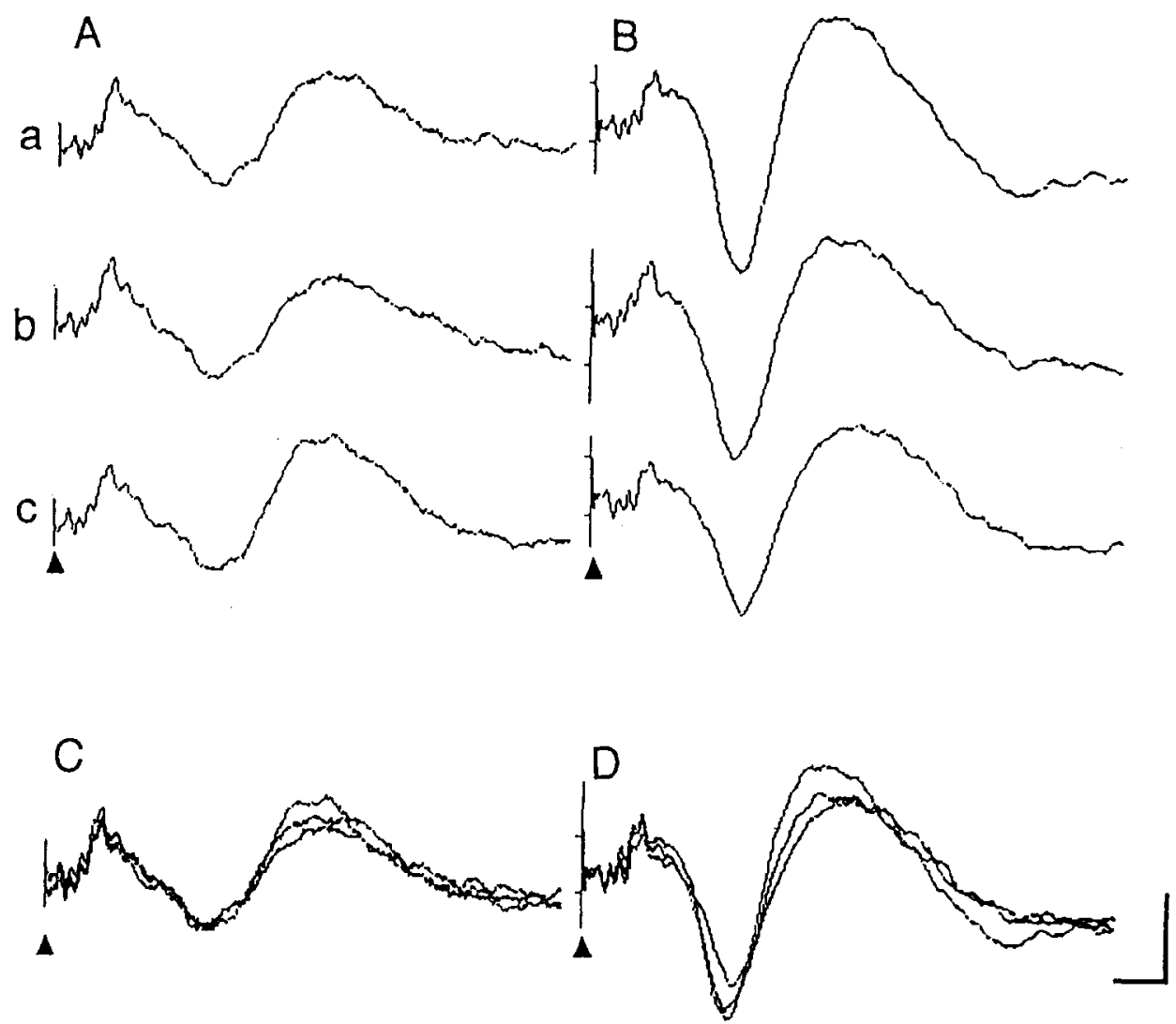

Fig. 2. Effects of tobacco cigarette smoking and abstinence on middle latency auditory evoked potentials (MLAEP) among smokers. The organization of this figure is similar to that of Fig. 1 for nonsmokers. However, group A potentials represent the averaged MLAEP from first $(a)$, second (b), and third (c) waves recorded for nine smokers averaged and abstinent for 6 to 15 hours. The averaged MLAEP for group B follow a 4-minute period of smoking one cigarette. The three tracings derived from each session were superimposed for the abstinence in $\mathbf{C}$ and after cigarette smoking in D. In contrast to Fig. $1, C$, in which the three separate potentials $a, b$, and $c$ over 9 to 10 minutes are very similar when superimposed, they are slightly less so for Fig. 2, C (ahstinence) and much more for Fig. 2, D (smoking). Note the robust Pa peaks after smoking a cigarette. The latency prolongation of Pa especially results in progressively less of an effect from the third as opposed to the first MLAEP, probably because of the declining nicotine blood levels or tachyphylaxis or both.

period of abstinence of smokers was considered to be 6 to 15 hours. Mean \pm SEM plasma nicotine concentrations at the beginning and end were $19.8 \pm 3.6$ and $28.2 \pm 3.69 \mathrm{ng} / \mathrm{ml}$ in the smoking session $(n=8)$ and $5.6 \pm 1.7$ and $3.8 \pm 1.4 \mathrm{ng} / \mathrm{ml}$ in the abstinent session $(n=7)$. Mean \pm SEM plasma cotinine levels at the beginning and end were $245.6 \pm 35.3$ and $256.2 \pm 29.8$ $\mathrm{ng} / \mathrm{ml}$ in the smoking session $(n=8)$ and $213.2 \pm 25.7$ and $214.2 \pm 26.4 \mathrm{ng} / \mathrm{ml}$ in the abstinent session $(n=7)$. Mean \pm SEM alveolar carbon monoxide levels at the beginning and end were $5.4 \pm 1.0$ and $7.0 \pm 0.92 \mathrm{ppm}$ in the smoking session $(n=9)$ and $1.7 \pm 0.52$ and 1.1 $\pm 0.35 \mathrm{ppm}$ in the abstinent sessions $(n=9)$.
Middle latency auditory evoked potentials in nonsmokers. Each MLAEP recording lasted about 2 minutes and was repeated until three reliable MLAEP waves were obtained for each subject. This may have partially confounded the results because changes may exist in auditory brain responses from repeated trials, as occurs with some other AEP components. ${ }^{24}$ However, both the smokers and nonsmokers were treated in the same manner.

The MLAEPs showed considerable between- and within-subject variability. Nevertheless, the MLAEPs had a more or less consistent pattern and allowed one to identify the components, including $\mathrm{Na}$ and $\mathrm{Pa}$ and 
A
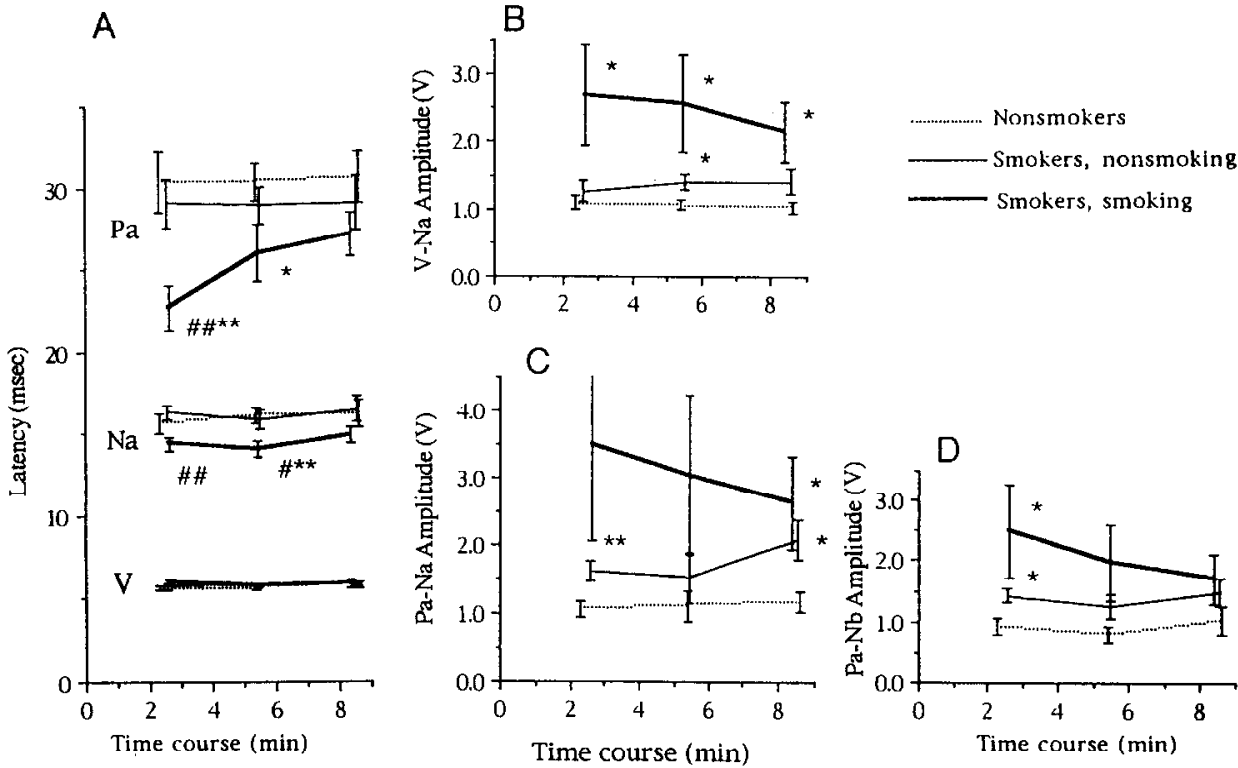

Fig. 3. Line graphs of latencies and peak-to-trough amplitudes of middle latency auditory evoked potentials in nonsmokers and smokers in the smoking and abstinent session. A, Time course of the latencies of positive peak V (lower panel), negative peak $\mathrm{Na}$ (middle panel), and positive peak $\mathrm{Pa}$ (upper panel). B, Time course of V-Na amplitude. C, Time course of Na-Pa amplitude. D, Time course of $\mathrm{Pa}-\mathrm{Nb}$ amplitude. The latencies and peak-to-trough amplitudes of the MLAEP components are plotted against time after actual or sham smoking. ${ }^{*} p<0.05,{ }^{* *} p<0.01$ compared with nonsmokers; $\# p<0.05$, \# $p<0.01$ compared with the abstinent session in smokers.

the early short latency components I through VI, for all subjects in both sessions. Potential Po could not be recorded reliably in all subjects and sessions. The $\mathrm{Nb}$ potential latency was sometimes difficult to determine in a small number of instances in which a rather blunted $\mathrm{Nb}$ peak with latencies as late as $50 \mathrm{msec}$ occurred. However, the $\mathrm{Nb}$ amplitude was more reliable because the line before and after this wave was almost isopotential or flat in most of the MLAEP. Therefore Nb amplitudes were used for further analysis in addition to the latencies and amplitudes of $\mathrm{Na}$ and $\mathrm{Pa}$.

Statistical analysis showed that none of the latencies or peak-to-trough amplitudes of the MLAEP components in nonsmokers differed significantly among the first, second, and third recordings either in the first or second session. There was no tendency toward change related to repetition of the procedures. These results as averaged waveforms are shown in Fig. 1. The averaged waveforms constructed from the nonsmokers' first, second, and third MLAEP waves both in the first session (Fig. 1, $A$ ) and second session (Fig. 1, $B$ ) show a similar highly reproducible pattern. As shown in Fig. 1, $C$, the two global averages were very replicable, indicating that two separate experimental sessions produced reproducible early and middle latency AEP in this group of nonsmokers. It was impressive that early and middle latency AEP among nonsmokers acquired in repeated trials or in separate sessions appeared to be independent and highly reproducible events.

Middle latency auditory evoked potentials after smoking and abstinence among cigarette smokers. Fig. 2 illustrates the averaged MLAEP of smokers. This average consisted of the first, second, and third replications in their abstinent (Fig. 2, A) and smoking (Fig. 2, B) sessions. These three tracings derived from the smoking or abstinent sessions were superimposed (Fig. 2, $C$ and $D$ ). As shown in this figure, the abstinent smokers repeatedly had mean MLAEPs that were similar throughout the observation period. In contrast, in the smoking session, the peaks in the averaged MLAEP had much shorter latencies and larger amplitudes than in the abstinent session. The latencies and amplitudes tended to recover consistently during the course of repeated observation. In the smoking sessions, the time between the end of the preceding smoking and start of the first recordings (mean \pm SEM) was 1 minute and 33 seconds \pm 32 seconds $(n=9)$. Observations were made up to 9 minutes and 


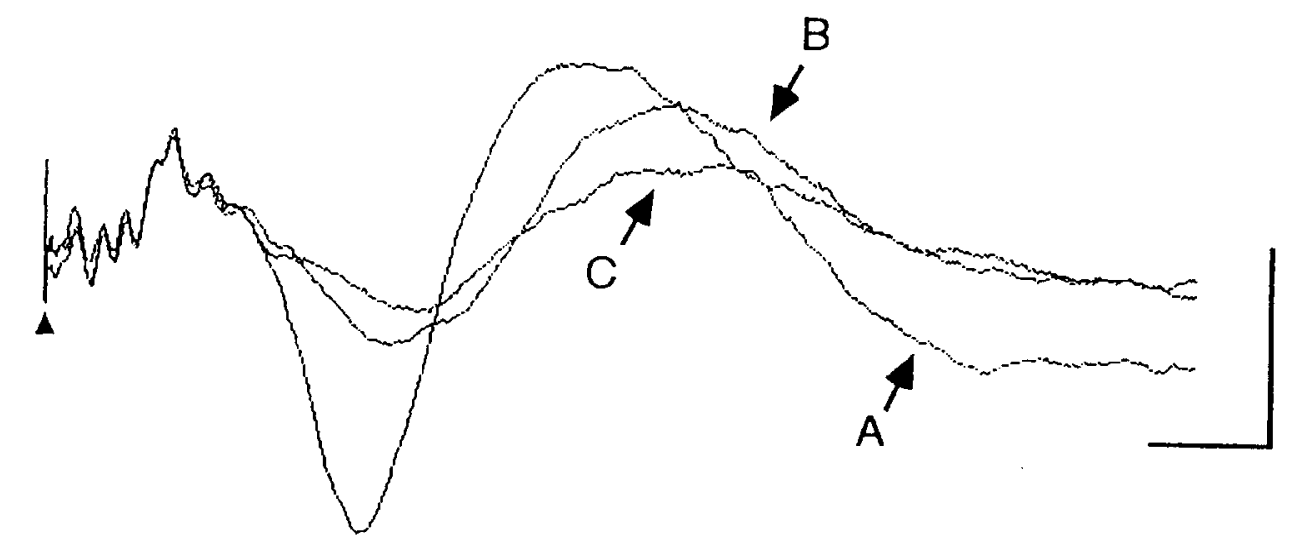

Fig. 4. Grand mean waveforms of the three middle latency auditory evoked potentials (MLAEP) obtained for smokers and nonsmokers. Tracing $A$, After cigarette smoking; tracing $B$, after partial abstinencc; tracing $C$, the first scssion of nonsmokers. The MLAEP waves were averaged for each subject group, and the mean MLAEP waves were further averaged over the first, second, and third recordings. The MLAEP appcar to be quite different in these three different sessions, in contrast to the early potentials with latencies less than $10 \mathrm{msec}$, which are more reproducible.

25 seconds \pm 31 seconds $(n=9)$ after each smoked cigarette. As shown in Fig. 3, the $\mathrm{Na}$ and $\mathrm{Pa}$ latencies were significantly (noncorrelated $t$ test $p<0.01$ ) shorter after smoking than abstinence. Similarly, the peak-to-trough amplitudes of MLAEP components (including V-Na [Fig. 3, B], Na-Pa [Fig. 3, C], and $\mathrm{Pa}-$ Nb [Fig. 3, D] amplitudes) tended to be larger in the smoking sessions than during the abstinent sessions or for nonsmokers.

To determine the global difference between MLAEP during smoking and abstinence, MLAEP obtained from all three recordings for each subject were averaged. When the averaged MLAEP over three repeated recordings for each subject were further averaged across all smokers, $\mathrm{Na}$ and $\mathrm{Pa}$ latencies were significantly ( $p<$ 0.05 ) shorter in the smoking than in the abstinent session. In agreement with this finding, the averages of the peak-to-trough amplitudes for $\mathrm{V}-\mathrm{Na}, \mathrm{Na}-\mathrm{Pa}$, and $\mathrm{Pa}$ $\mathrm{Na}$ over the three repeated measures tended to be larger in the smoking than in the abstinent session.

Fig. 4 illustrates the global MLAEP averaged over the three MLAEP obtained throughout the three observation periods in the smoking (tracing A) and abstinent sessions. The MLAEP averaged over about 9 minutes after cigarette smoking for all smokers had $\mathrm{Na}$ and $\mathrm{Pa}$ peaks with shorter latencies and larger amplitudes than the abstinent session or for nonsmokers. The early AEPs with latencies less than $10 \mathrm{msec}$ appeared to be quite similar to each other, in contrast to the later MLAEP components. The early portion of the AEP is considered to represent brainstem AEP, which in this figure appear somewhat different from published early responses because of different conditions and a compressed time $x$-axis.

Middle latency auditory evoked potentials of smokers just after smoking compared with nonsmokers. Fig. 4 summarizes in graphic form the comparison between MLAEP for smokers after cigarette smoking $(n=9)$ and nonsmokers (first session, $n=10$ ). $\mathrm{Na}$ and $\mathrm{Pa}$ latencies were significantly $(p<0.01)$ shorter after cigarette smoking than for nonsmokers. V-Na, Na-Pa, and $\mathrm{Pa}-\mathrm{Nb}$ peak-to-trough amplitudes were also significantly $(p<0.05)$ larger after cigarette smoking than those for nonsmokers. Differences in these MLAEP indexes between smokers under smoking conditions and nonsmokers tended to be smaller in the course of three repeated recording periods. However, the averages of the three recordings of $\mathrm{Na}$ and $\mathrm{Pa}$ latencies and V-Na peak-to-trough amplitude differed significantly $(p<0.05)$ between the smokers' smoking and nonsmokers' sessions. As shown in Fig. 4, the global mean wave resulting from the three MLAEP in the smoking session for smokers (tracing A) had $\mathrm{Na}$ and $\mathrm{Pa}$ peaks with shorter latencies and larger amplitude than those in the nonsmokers' first session (tracing C). Analyses also were performed with other possible nonsmoker combinations, such as the second session or randomized arbitrary nonsmoker combinations in which data from half of the nonsmokers were collected from either the first and second sessions of nonsmokers versus smoker group combinations. Similar results were obtained. 
Middle latency auditory evoked potentials for partially abstinent smokers compared with nonsmokers. The data are summarized in Fig. 4. The latencies of MLAEP tended to be slightly shorter for the abstinent smokers than the nonsmokers, but the differences between these two conditions were not significant. However, $\mathrm{V}-\mathrm{Na}$ and $\mathrm{Na}-\mathrm{Pa}$ peak-to-trough amplitudes were significantly $(p<0.05)$ larger for abstinent smokers than for nonsmokers. The Pa-Nb peak-totrough amplitude also tended to be larger among abstinent smokers. Averages of the $\mathrm{V}-\mathrm{Na}$ and $\mathrm{Na}-\mathrm{Pa}$ peakto-trough amplitudes over three recordings were significantly $(p<0.05)$ larger among abstinent smokers $(n=9)$ than nonsmokers $(n=10)$. Additional analyses of other possible combinations of nonsmoker and abstinent smoker groups yielded similar results.

\section{DISCUSSION}

There is no question that MLAEP differ among smokers compared with nonsmokers, and early latency AEP do not. In addition, there are significant differences between abstinent smokers and nonsmokers. The relevance of these findings can be appreciated only if one appreciates the neurophysiologic meaning of MLAEP, especially $\mathrm{Na}, \mathrm{Pa}$, and $\mathrm{Nb}$. These waves were recorded from the vertex of the scalp. Although the neuroanatomic pathways of MLAEP are not completely clarified, many studies suggest that wave $\mathrm{Pa}$ appears to involve mainly subcortical structures, including the thalamus or thalamocortical projections or both. 25,26 The peak $\mathrm{Na}$ has been shown to originate in the midbrain region and project to the medial geniculate body and then to the temporal lobe. ${ }^{27}$ The early components of the AEP reflect rapid transmission in subcortical structures en route to the primary sensory cortex. ${ }^{28}$ MLAEP involve neuronal activities that originate in the primary auditory pathways and nonprimary sensory pathways. ${ }^{26}$ Primary auditory pathways in the brain have fine frequency tuning and precise time-locked transmission. The nonprimary pathways are multimodal, broadly tuned, and reflect attention and integration of multimodality information. ${ }^{29}$ MLAEP recorded in the midline are associated more with the nonprimary neuronal areas in animals. ${ }^{30}$ Animal studies have shown that these components receive critical input from the mesencephalic reticular formation ${ }^{26}$ and that the amplitudes of these components reflect the subject's state of arousal, ${ }^{16,17}$ as described earlier.

Positive effects of tobacco cigarette smoking on middle latency auditory evoked potentials. Repeatedly recorded MLAEPs for smokers after cigarette smoking were compared with those of nonsmokers. There were some differences in the experimental conditions between the two groups. Smokers underwent blood sampling and cigarette smoking that were repeated bcfore and during the laboratory session. Nonsmokers underwent neither. However, MLAEP among smokers decremented rather rapidly after cigarette smoking, suggesting either a small influence of venous blood sampling on MLAEP or the rapid decline in plasma nicotine concentrations resulting from a short half-life. Furthermore, the early components of AEP with latencies less than $10 \mathrm{msec}$ appeared to be relatively unaffected, in contrast to the later MLAEP. $\mathrm{Na}$ and $\mathrm{Pa}$ latencies among smokers during the smoking session were significantly $(p<0.01)$ shorter than among nonsmokers. Although latencies for MLAEP do not directly reflect the time for auditory sensory information to be transmitted to the auditory cortex, the foregoing finding suggests that complex sensory information processing between the brainstem and thalamocortical reverberations is more rapid among smokers after smoking than among nonsmokers, especially for peak $\mathrm{Pa}$. In agreement with the latency changes for MLAEP, V-Na, $\mathrm{Na}-\mathrm{Pa}$, and $\mathrm{Pa}-\mathrm{Nb}$ peak-to-trough amplitudes also were larger among smokers after smoking than among nonsmokers, suggesting association with a higher arousal level among smokers when repeatedly smoking than among nonsmokers. The $\mathrm{Pa}$ amplitude reflects stimulation of the mesencephalic reticular formation and correlates with the arousal level. Therefore our results suggest that tobacco smoking may induce a higher arousal level, presumably caused by activation of the mesencephalic reticular formation.

The marked differences in MLAEP between smokers after smoking and nonsmokers decreased over the course of the repeated recordings in a 9- to 10-minute period. Because repetition of recording by itself did not produce any appreciable change in MLAEP in the control subjects, this time-dependent reduction of MLAEP appears to be associated with rapid decay in plasma nicotine concentrations after smoking one cigarette. ${ }^{19,20}$ Pharmacodynamic adaptation or tachyphylaxis may also play a role. Cigarette smoking appears to enable smokers to undergo, in relatively a short period of 10 minutes or so, various arousal levels. Although higher arousal levels do not necessarily result in better overall mental functioning, ${ }^{31}$ our results suggest that smokers take advantage of the stimulating effects of nicotine. Better mental functioning or an optimal arousal level may yield subjective pleasure, inasmuch as targeting an optimal arousal level seems to be self-rewarding. ${ }^{32}$ Thus the positive effects of smoking cigarettes on sensory information processing, along 
with an increase in arousal level (exemplified by changes in MLAEP), may be associated with the positive reinforcing properties of nicotine.

Changes in MLAEP indexes induced by tobacco smoking appeared to be more closely correlated with plasma concentration of nicotine, roughly showing concentration-dependent MLAEP responses to nicotine across smokers, rather than with inhalation behavior.

Negative effect of tobacco abstinence on middle latency auditory evoked potentials. The MLAEP in the smoking and abstinent sessions of smokers were compared. The abstinent session was designed so that the smokers were supposed to have been deprived of both nicotine and smoking for at least 6 to 15 hours. Smoking sessions were designed to resemble the smoker's usual smoking behavior. Smokers deprived of smoking for at least 6 hours had mean MLAEPs of smaller amplitude and longer latency than when they smoked. Robust peaks in MLAEP in the smoking sessions showed time-dependent depression over 9 to $10 \mathrm{~min}$ utes. On the other hand, the latencies and amplitudes of MLAEP of smokers after partial abstinence and nonsmoking healthy subjects were relatively constant throughout the observation period. Furthermore, the early AEP with latencies less than $10 \mathrm{msec}$ appeared quite replicable for all three groups, in contrast to the later MLAEP. These results suggest relatively poor information processing in the responsible pathways above the brainstem, including the auditory and association cortex, among abstinent smokers compared with those repeatedly smoking cigarettes. Such poor functioning after partial abstinence may discourage smokers from smoking abstinence.

Depression of MLAEP among smokers after partial abstinence is not necessarily evidence of physical dependence with associated neuronal changes. An unexpected finding was that compared with nonsmokers, some tobacco smokers had MLAEP indexes that suggested a more stimulated brain state even after abstinence. A few of the smokers reported drowsiness during their abstinent session. The 6- to 15-hour period of tobacco abstinence used in this study may not be long enough to produce a full nicotine withdrawal syndrome for most smokers. Our results agree with those in other reports in which withdrawal syndromes after tobacco abstinence of 1 hour to 21 days were examined. ${ }^{5,15,33-35}$ Because plasma nicotine levels indicated that two of the nine smokers were probably not completely abstinent, our results may be attributable to lack of compliance and lack of truthfulness on the part of smokers. ${ }^{22,23}$

Behavioral arousal appears to result from a dynamic equilibrium between the reticular activating system, which provides a tonic background of neocortical responsiveness, and the limbic system, which focuses attention on relevant factors. ${ }^{31}$ Auditory information is known to be transmitted to limbic structures, such as the hippocampus, and to the basal forebrain. Nicotine is known to act on these structures to modulate cholinergic gating of auditory information. ${ }^{36,37}$ Animal studies have shown that the rewarding effects of nicotine involve brain areas closely associated with these structures. ${ }^{38} \mathrm{~A}$ wider range of AEP, especially studied in combination with behavioral end points, may provide better insight into the motivation for tobacco smoking and its relation to neuropsychologic factors such as arousal, attention, and cognition. Nevertheless, the results of this study support the notion that tobacco smoking is maintained by its positive effect on information processing and avoidance of its relative reduction after short periods (hours) of tobacco abstinence.

\section{References}

1. McKennell AC. Smoking motivation factors. Br J Soc Clin Psychol 1970;9:8-22.

2. Church RE. Smoking and the human EEG. In: Ney T, Gale A, editors. Smoking and human behavior. New York: John Wiley; 1989. p. 115-40.

3. Clarke PBS. The central pharmacology of nicotine: electrophysiological approaches. In: Wonnacott S, Russell MAH, Stolerman IP, editors. Nicotine psychopharmacology: molecular, cellular and behavioural aspects. New York: Oxford University Press; 1990. p. 158-93.

4. Knott VJ, Venables PH. EEG alpha correlates of nonsmokers, smokers, smoking, and smoking deprivation. Psychophysiology 1977;14:150-6.

5. Pickworth WB, Herning RI, Henningfield JE. Spontaneous EEG changes during tobacco abstinence and nicotine substitution in human volunteers. J Pharmacol Exp Ther 1989;251:976-82.

6. Domino EF, Riskalla M, Zhang Y, Kim E. Effects of tobacco smoking on the topographic EEG II. Prog Neuropsychopharmacol Biol Psychiatry 1992;16:463-82.

7. Domino EF, Kadoya C, Matsuoka S. Effects of tobacco smoking on the topographic electroencephalogram. In: Domino EF, editor. Brain imaging of nicotine and tobacco smoking. Ann Arbor (MI): NPP Books; 1995. p. 253-61.

8. Knott VJ, Hooper C, Lusk-Mikkelsen S, Kerr C. Variations in spontaneous brain electrical (EEG) topography related to cigarette smoking: acute smoking, drug comparisons, cholinergic transmission, individual differences and psychopathology. In: Domino EF, editor. Brain imaging of nicotine and tobacco smoking. Ann Arbor (MI): NPP Books; 1995. p. 167-89.

9. Hobson J, Brazier MAB. The reticular formation revisited: specific function for a nonspecific system. IBRO monograph 6. New York: Raven Press; 1980. p. 1-552. 
10. Knapp DE, Domino EF. Action of nicotine on the ascending reticular activating system. Int $\mathbf{J}$ Neuropharmacol 1962;1:333-51.

11. Domino EF, Yamamoto K. Nicotine: effect on the sleep cycle of the cat. Science 1965;150:637-8.

12. Yamamoto $\mathrm{K}$, Domino EF. Nicotine-induced EEG and behavioral arousal. Int J Neuropharmacol 1965;4: 359-73.

13. Kawamura H, Domino EF. Differential actions of $m$ and $n$ cholinergic agonists on the brainstem activating system. Int J Neuropharmacol 1969;8:105-15.

14. Domino EF. Neuropsychopharmacology of nicotine and tobacco smoking. In: Dunn WL Jr, editor. Smoking behavior: motives and incentives. Washington (DC): VH Winston; 1973. p. 5-31.

15. Pickworth WB, Heishman SJ, Henningfield JE. Relationships between EEG and performance during nicotine withdrawal and administration. In: Domino EF, editor. Brain imaging of nicotine and tobacco smoking. Ann Arbor (MI): NPP Books; 1995. p. 275-87.

16. Osterhammel PA, Shallop JK, Terkildsen K. The effect of sleep on the auditory brainstem response (ABR) and the middle latency response. Scand Audiol 1985;14:47-50.

17. Jones LA, Baxter RJ. Changes in the auditory middle latency responses during all-night sleep recording. $\mathrm{Br} \mathrm{J}$ Audiol 1988;22:279-85.

18. Kraus N, Ozdamar O, Hier D, Stein L. Auditory middle latency responses (MLRs) in patients with cortical lesions. Electroencephalogr Clin Neurophysiol 1982;54:275-87.

19. Benowitz NL, Kuyt F, Jacob P III. Circadian blood nicotine concentration during cigarette smoking. Clin Pharmacol Ther 1982;32:758-64.

20. Benowitz NL, Porchet H, Jacob P III. Pharmacokinetics of nicotine. In: Wannacott $\mathrm{S}$, Russell MAH, Stolerman IP, editors. Nicotine psychopharmacology: molecular, cellular, and behavioral aspects. Oxford, England: Oxford University Press; 1990. p. 112-57.

21. Hariharan N, Van Noord T, Greden JF. A high-performance liquid-chromatographic method for routine simultaneous determination of nicotine and cotinine in plasma. Clin Chem 1988:34:724-9.

22. Apseloff G, Ashton HM, Friedman H, Gerber N. Pharmacoepidemiology and drug utilization: the importance of measuring cotinine levels to identify smokers in clinical trials. Clin Pharmacol Ther 1994;56:460-2.
23. Reidenberg MM. Some smokers lie [editorial]. Clin Pharmacol Ther 1994;56:355.

24. Knott VJ. Acute effects of tobacco on human brain stem evoked potentials. Addict Behav 1987;12:375-9.

25. Kennely JH, Kilroy P, Paccioretti D, McLean DR. Neurologic, audiologic, and electrophysiologic sequelae of bilateral temporal lobe lesions. Arch Neurol 1987;44:982-7.

26. Kraus N, McGee T, Niclo T. Reticular formation influences on primary and non-primary auditory pathways as reflected by the auditory middle latency response. Brain Res 1992;587:186-94.

27. Hashimoto I. Auditory evoked potentials from the human mid-brain: slow brainstem responses. Electroencephalogr Clin Neurophysiol 1982;53:652-7.

28. Chiappa K. Evoked potentials in clinical medicine. 2nd ed. New York: Raven Press; 1990. p. 1-647.

29. Winter JA. The human medial geniculate body. Hear Res $1984 ; 15: 225-47$

30. Kraus N, McGee T. Clinical implication of primary and nonprimary pathway contributions to the middle latency response generating system. Ear Hear 1993;14:36-48.

31. Routtenberg A. The two-arousal hypothesis: reticular formation and limbic system. Psychol Rev 1968;75:51-80.

32. Ashton H, Golding JF. Motivation and models. In: Ney T, Gale A, eds. Smoking and human behavior. New York: John Wiley; 1909. p. 21-56.

33. Hatsukami DK, Hughes JR, Pickens RW, Svikes D. Tobacco withdrawal symptoms: an experimental analysis [published erratum appears in Psychopharmacology 1992;108:390]. Psychopharmacology (Berl) 1984;84:231-6.

34. Hughes JR, Hatsukami D. Signs and symptoms of tobacco withdrawal. Arch Gen Psychiatry 1986;43:289-94.

35. Snyder RF, Davis FC, Henningfield JE. The tobacco withdrawal syndrome: performance decrements assessed on a computerized test battery. Drug Alcohol Depend 1989:23:259-66

36. Luntz-Leybman V, Bickford PC, Freedman R. Cholinergic gating of response to auditory stimuli in rat hippocampus. Brain Res 1992;587:130-6.

37. Miller CL, Freedman R. Medial septal neuron activity in relation to an auditory sensory gating paradigm. Neuroscience 1993;55:373-80.

38. Ando K, Miyata H, Hironaka N, Tsuda T, Yanagita T. The discriminative effects of nicotine and their central sites in rats. Jpn J Psychopharmacol 1993;13:129-36. 